\section{Efecto antibacteriano in vitro del extracto de Stevia rebaudiana sobre Streptococcus sanguinis y Actinomyces viscosus, bacterias iniciadoras en la formación de biopelícula dental}

\author{
In vitro antibacterial effect of Stevia \\ rebaudiana extract on Streptococcus \\ sanguinis and Actinomyces viscosus, \\ starter bacterias in dental biofilm formation
}

\begin{abstract}
Resumen
Objetivo: Determinar el efecto antibacteriano del extracto de Stevia rebaudiana (S. rebaudiana) frente a Streptococcus sanguinis (S. sanguinis) y Actinomyces viscosus (A. viscosus). Métodos: Se desarrolló la prueba de sensibilidad en placa de agar con discos, para lo cual se cultivaron las cepas de $S$. sanguinis y A. viscosus en placas de agar tripticasa soya (TSA) y agar sangre respectivamente, incubando a $37^{\circ} \mathrm{C}$ por 48 horas a S. sanguinis y por 7 días en condiciones de anaerobiosis a $A$. viscosus. Las cepas bacterianas fueron estandarizadas a una escala de 0,5 de Mc Farland, y tomando inóculos de $100 \mu \mathrm{L}$ fueron sembradas en diez placas de agar sangre y TSA, luego sobre cada placa se colocaron los discos de papel secante de $6 \mathrm{~mm}$ de diámetro de forma equidistante, cargados con $10 \mu \mathrm{L}$ de las diferentes concentraciones del extracto, para luego ser incubados. Resultados: Las concentraciones de $15,30,50,60$ y $120 \mathrm{mg} / \mathrm{ml}$ presentaron un halo de inhibición promedio de $6,8 \pm 0,258 ; 8,2 \pm 1,15 ; 8,2 \pm 1,13 ; 8,3 \pm 0,823 ; 8,1 \pm 0,80 \mathrm{~mm}$ respectivamente, para las bacterias de $S$. sanguinis. Las concentraciones de $15,30,50,60$ y $120 \mathrm{mg} / \mathrm{ml}$ presentaron un halo de inhibición promedio de 7,2 $\pm 0,75 ; 9,65 \pm 2,15 ; 9,20 \pm 2,03 ; 7,95 \pm 1,09 ; 8,05 \pm 0,96$ mm respectivamente, para las bacterias de $A$. viscosus. La prueba de Kruskal Wallis determinó que existe diferencia estadísticamente significativa con $p<0,05$ de los promedios entre las concentraciones de $A$. viscosus. Conclusiones: El extracto de $S$. rebaudiana no presenta efecto antibacteriano para $S$. sanguinis, pero si presenta efecto antibacteriano sobre A. viscosus para las concentraciones de 30 y $50 \mathrm{mg} / \mathrm{ml}$.
\end{abstract}

Palabras clave: Antibacteriano; Actinomyces viscosus; Biopelícula dental; Stevia; Streptococcus sanguis.
ISSN: 1560-9111

Artículo Original

Katherine Brañez Reyes ${ }^{1, a}$, Donald

Ramos-Perfecto ${ }^{2, a, b}$, Américo Castro Luna ${ }^{3, c}$,

César Piscoche Botello 1,a, Darío Dávila

Paredes ${ }^{4, d}$, Juan Celidonio Ruiz Macedo 4,d

${ }^{1}$ Facultad de Odontología. Universidad Nacional Mayor de San Marcos. Lima, Perú.

2 Departamento Académico de Ciencias Básicas. Facultad de Odontología. Universidad Nacional Mayor de San Marcos. Lima, Perú. ${ }^{3}$ Facultad de Farmacia y Bioquímica. Universidad Nacional Mayor de San Marcos. Lima, Perú.

${ }^{4}$ Centro de Investigaciones de Recursos

Naturales, CIRNA. Universidad Nacional de la Amazonia Peruana. Loreto, Perú.

${ }^{a}$ Cirujano Dentista.

b Magíster en Microbiología.

${ }^{c}$ Doctor en Farmacia y Bioquímica.

${ }^{d}$ Ingeniero Forestal.

\section{Correspondencia:}

Katherine Brañez Reyes

Correo electrónico: katherine.brañez@gmail. com

Calle Salvador 770 Nocheto, Santa Anita, Lima 1 , Perú.

\section{Coautores:}

Donald Ramos Perfecto dramosp@unmsm.edu.pe Américo Castro Luna acastrol@unmsm.edu.pe César Piscoche Botello cpiscocheb@unmsm.edu.pe Darío Dávila Paredes davilaparedesdario@yahoo.es Juan Celidonio Ruiz Macedo juaceruma@yahoo.es

Conflicto de intereses: Los autores declaran no tener conflictos de interés.

Fuente de financiamiento: Con apoyo del Vicerrectorado de Investigación de la Universidad Nacional Mayor de San Marcos

Fecha de recepción: 03/10/17 Fecha de aceptación: 27/02/18

( ) Los autores. Este artículo es publicado por la revista Odontología Sanmarquina de la Facultad de Odontología, Universidad Nacional Mayor de San Marcos. Este es un artículo de acceso abierto, distribuido bajo los términos de la licencia Creative Commons Atribucion - No Comercia_Compartir Igual 4.0 Internacional. (http://creativecommons.org/licenses/by-nc-sa/4.0/) que permite el uso no comercial, distribución y reproducción en cualquier medio, siempre que la obra original sea debidamente citada. 


\begin{abstract}
Objective: To determine the antibacterial effect of Stevia rebaudiana extract (S. rebaudiana) against Streptococcus sanguinis ( $S$. sanguinis) and Actinomyces viscosus (A. viscosus). Methods: The plaque agar sensitivity test was developed with discs, for which strains of S. sanguinis and $A$. viscosus were grown on trypticase soy agar plates (TSA) and blood agar, respectively, incubating at $37^{\circ} \mathrm{C}$. for 48 hours to $S$. sanguinis and for 7 days under conditions of anaerobiosis to $A$. viscosus. The bacterial strains were standardized at a scale of 0.5 of Mc Farland, and taking inocula of $100 \mu \mathrm{L}$ were seeded in ten plates of blood agar and TSA, then in each plate were placed discs of $6 \mathrm{~mm}$ diameter in equidistant form, loaded with $10 \mu \mathrm{L}$ of different concentrations of the extract, to incubate. Results: The concentrations of $15,30,50,60$ and $120 \mathrm{mg} / \mathrm{ml}$ presented an average inhibition halo of $6.8 \pm 0.258 ; 8.2 \pm 1.15 ; 8.2 \pm 1.13 ; 8.3 \pm 0.823 ; 8.1 \pm 0.80 \mathrm{~mm}$ respectively, for the bacterium S. sanguinis. The concentrations of $15,30,50,60$ and $120 \mathrm{mg} / \mathrm{ml}$ showed an average inhibition halo of $7.2 \pm 0.75$; $9.65 \pm 2.15 ; 9.20 \pm 2.03$; $7.95 \pm 1.09 ; 8.05 \pm 0.96 \mathrm{~mm}$ respectively, for the bacterium A. viscosus. The Kruskal Wallis test determined that there is a statistically significant difference with $p<0.05$ of the averages between the concentrations of $A$. viscosus. Conclusions: The extract of $S$. rebaudiana does not have an antibacterial effect for $S$. sanguinis, but has an antibacterial effect on $A$. viscosus for concentrations of 30 and $50 \mathrm{mg} / \mathrm{ml}$.
\end{abstract}

Keywords: Anti-bacterial agents; Actinomyces viscosus; Dental plaque; Stevia; Streptococcus sanguis.

\section{Introducción}

Estudios epidemiológicos del Ministerio de Salud del Perú reportan que la prevalencia de caries dental en la población general es del $90,4 \%{ }^{1}$ y según la Organización Mundial de la Salud (OMS) ${ }^{2}$, el 60\%-90\% de los escolares y casi el 100\% de los adultos tienen caries dental en todo el mundo, afirmando que las principales afecciones bucales como caries dental y enfermedad periodontal constituyen un importante problema de salud debido a su alta prevalencia e incidencia en la salud general de la población mundial ${ }^{3}$. La caries dental es una enfermedad multifactorial que se caracteriza por la desmineralización progresiva de las porciones inorgánicas del diente y el deterioro posterior de las porciones orgánicas de este ${ }^{4}$. Es un proceso destructivo que se origina por la acción de los microorganismos que forman parte de la biopelícula dental ${ }^{5,6}$. Esta es una enfermedad localizada que resulta de los procesos metabólicos de la biomasa microbiana en contacto con la superficie dental y la dieta, que proporcionan los requerimientos nutricionales y por lo tanto energéticos a los microorganismos de la microbiota bucal 7 . En relación a los microorganismos se ha determinado que existe una flora bacteriana iniciadora en la formación de la biopelícula dental, la cual es considerada como el mayor factor asociado a la aparición de la caries dental, entre los cuales están Streptococcus sanguinis (S. sanguinis) y Actinomyces viscosus (A. viscosus).

Streptococcus sanguinis es un microorganismo que forma parte del ecosistema oral, pertenece al grupo de Streptococcus viridans, muy asociado a patologías como la endocarditis bacteriana. En relación a patologías bucales, es el iniciador en la formación de la biopelícula dental o placa dental, complejo asociado a la caries dental y enfermedades periodontales. Este microorganismo presenta como unidad formadora un estructura llamada coco de aproximadamente $0,6 \mu \mathrm{m}$ de diámetro, que se dis- pone en cadenas medianas o largas. Son Gram positivo, catalasa negativa y anaerobios facultativos. Su genoma es un ADN circular de 2388435 pb. Entre sus factores de virulencia destacan sus fimbrias y adhesinas, estructuras que le permiten gran adherencia a la película adquirida dental, así como la producción de glucosiltransferasas responsables en la formación de glucanos, compuestos que van a permitir mayor adhesión de otros microorganismos ${ }^{8-10}$.

Actrinomyces viscosus, es una bacteria Gram positiva antiguamente llamada Odontomyces viscosus, morfológicamente es un bacilo que puede presentar forma curva, en barra, filamentosa larga o corta observándose por microscopia como formas ramificadas. Sus colonias son de un color blanco pálido brillante, circulares y de borde entero, esto es típico en un medio de agar sangre. Su factor de virulencia más resaltante son las fimbrias, como la fimbria tipo I, la cual tiene afinidad por las proteínas ricas en prolina y estaterina, así como por la superficie del esmalte dental a través de un mecanismo adhesina-receptor. Producen polisacáridos intracelulares y extracelulares de alto peso molecular a partir de sacarosa, que por medio de exoenzimas van a formar mútanos, dextranos y lévanos, siendo los mútanos compuestos que tienen gran función adhesiva y los otros dos usados como reserva alimenticia ${ }^{10,11}$.

Las enfermedades bucales de mayor prevalencia son causadas por un desequilibrio en las poblaciones bacterianas que están presentes de forman natural y que ayudan a mantener el estado normal de la cavidad bucal ${ }^{12}$. En apoyo al control de las enfermedades bucales, varias plantas medicinales de nuestro medio están siendo estudiadas como el Erythroxilum novogranatense var truxlense (hoja de coca) y Plantago major (llantén), evaluando su acción inhibitoria sobre el crecimiento de bacterias y hongos ${ }^{13}$. Otros estudios han analizado el efecto de la 
Camellia sinensis (té verde) en la formación de la placa bacteriana por Streptococcus mutans (S. mutans) ${ }^{14}$.

Stevia rebaudiana (S. rebaudiana) es un producto natural con propiedades antihiperglucemico, hipotensor, cardiotónico, así como un edulcorante que podría reemplazar al azúcar refinada muy utilizada en nuestros días. Esta planta es cultivada en nuestro país desde hace una década y es perfectamente adaptable a las regiones tropicales y subtropicales del Perú, como en las regiones de Cajamarca, Amazonas, San Martin, Ucayali y Apurímac ${ }^{15}$.

El estudio desarrollado tiene por objetivo determinar el efecto antibacteriano del extracto de $S$. rebaudiana, sobre dos bacterias consideradas iniciadoras de la biopelícula dental, como son $S$. sanguinis y $A$. viscosus

\section{Métodos}

El estudio fue de tipo experimental, prospectivo e in vitro; se realizó previa aceptación de la metodología del estudio por el Instituto de Investigación Estomatológica de la Facultad de Odontología de la Universidad Nacional Mayor de San Marcos (UNMSM).

Se utilizaron las cepas de $S$. sanguinis ATTC 10556 y $A$. viscosus ATTC 15985, las cuales pasaron un control de calidad en su obtención, como en su activación.

Las concentraciones de $15 \mathrm{mg} / \mathrm{ml}, 30 \mathrm{mg} / \mathrm{ml}, 50 \mathrm{mg} / \mathrm{ml}$, $60 \mathrm{mg} / \mathrm{ml}$ y $120 \mathrm{mg} / \mathrm{ml}$, del extracto hidro-alcoholico de Stevia rebaudiana se prepararon en el laboratorio de la Facultad de Farmacia y Bioquímica de la UNMSM.

Una vez obtenido el extracto y las cepas se procedió a desarrollar la prueba de sensibilidad, difusión en agar con disco.

Prueba de sensibilidad de difusión en placa de agar con disco. La activación de la cepas se realizó según microorganismo, $S$. sanguinis en medios de agar tripticasa soya (TSA) por $24-48$ horas a $37^{\circ}$ con $\mathrm{CO}$ al $5 \%$ y $A$. viscosus en agar sangre por 7 días en anaerobiosis, a 37 ${ }^{\circ} \mathrm{C}$. Luego de la activación de las cepas, se determinó para ambas cepas un nivel de turbidez de 0,5 de Mc Farland, se sembró por diseminación un inóculo de 100 $\mu \mathrm{l}$ de la dilución en agar TSA y sangre, seguidamente colocamos los discos conteniendo $10 \mu \mathrm{l}$ de las diferentes concentraciones de $S$. rebaudiana $(15 \mathrm{mg} / \mathrm{ml}, 30 \mathrm{mg} / \mathrm{ml}$, $50 \mathrm{mg} / \mathrm{ml}, 60 \mathrm{mg} / \mathrm{ml}$ y $120 \mathrm{mg} / \mathrm{ml}$ ), colocando un disco para el control positivo (clorhexidina al $0,12 \%$ ) y otro para el control negativo (etanol $96^{\circ}$ ). Posteriormente se procedió a incubar a $37^{\circ} \mathrm{C}$ por 48 horas en medio $\mathrm{CO}_{2}$ al 5\% (S. sanguinis) y 7 días en anaerobiosis ( $A$. viscosus). La lectura se realizó pasado el tiempo de incubación, en función a la medición de los diámetros de los halos de inhibición del crecimiento de los microorganismos (HICM) confrontando los resultados con la escala de Duraffourd: sensibilidad nula () si el HICM fue inferior o igual a $8 \mathrm{~mm}$; sensibilidad límite (sensible $=+$ ) de 9 a $14 \mathrm{~mm}$; sensibilidad media (muy sensible $=++$ ) de 15 a $19 \mathrm{~mm}$ y sumamente sensible $(\mathrm{S} . \mathrm{S}=+++)$ si fue igual o superior a $20 \mathrm{~mm}$.

\section{Resultados}

Los resultados mostraron que el extracto de $S$. rebaudiana tiene efecto antibacteriano in vitro sobre $A$. viscosus, siendo las medias de los diámetros de los halos de inhibición $9,65 \pm 2,15 ; 9,2 \pm 2,03 ; 8,05 \pm 0,96,7,95 \pm 1,09 y$ $7,2 \pm 0,75 \mathrm{~mm}$ correspondiendo el mayor promedio para la concentración de $30 \mathrm{mg} / \mathrm{ml}$ y la menor concentración para la de $15 \mathrm{mg} / \mathrm{ml}$ (Figura 1).

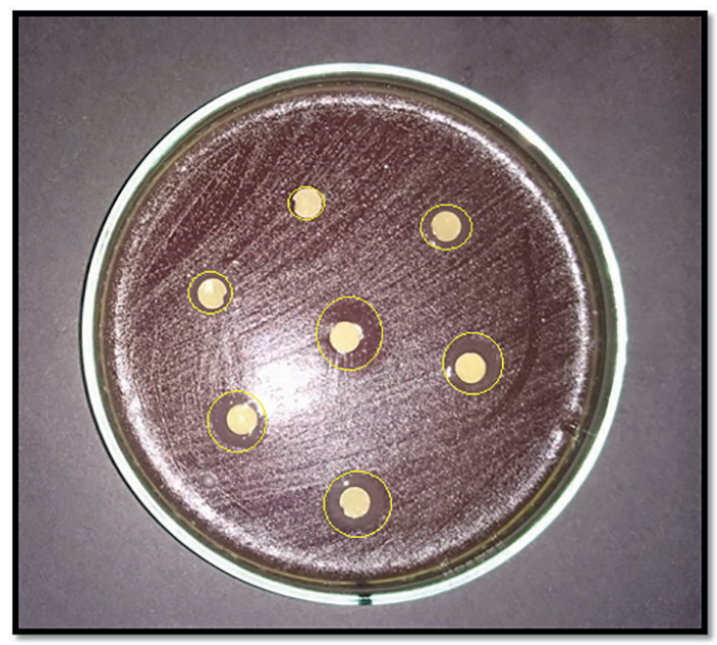

Figura 1. Halos de inhibición del extracto de S. rebaudiana a diferentes concentraciones sobre $A$. viscosus, en agar sangre

En la Figura 1 se puede apreciar que el efecto antibacteriano de $S$. rebaudiana sobre $A$. viscosus no es directamente proporcional a su concentración. El análisis estadístico mediante la prueba de Kruskall-Wallis indicó que existen diferencias significativas $(p=0,02)$ entre el efecto antibacteriano de las diferentes concentraciones de $S$. rebaudiana (Figura 2).

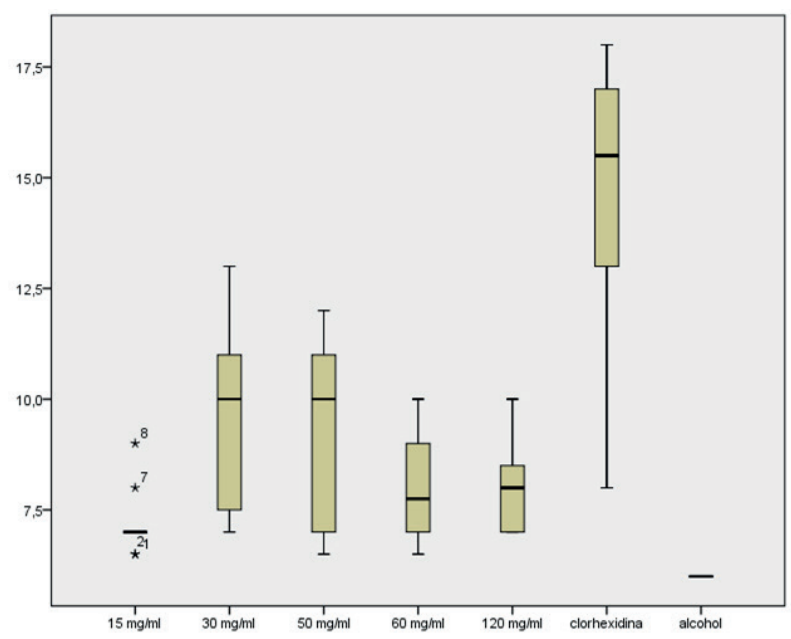

Figura 2. Resultados de los halos de inhibición en $\mathrm{mm}$ sobre $A$. viscosus causados por las diferentes concentraciones de Stevia rebaudiana, como de los controles positivo y negativo

En relación al efecto antibacteriano del extracto de $S$. rebaudiana sobre $A$. viscosus, se obtuvo que los halos de inhibición del crecimiento bacteriano que se encontraron en el rango de sensibilidad límite $(9-14 \mathrm{~mm})$ correspondieron a la formación de 19 halos (38\%) mientras 
que 31 halos $(62 \%)$ tuvieron sensibilidad nula ( $8 \mathrm{~mm})$ (según la escala de Duraffourd).

Los resultados mostraron que el extracto de $S$. rebaudiana no tiene actividad antibacteriana in vitro sobre $S$. sanguinis, siendo las medias de los diámetros de los halos de inhibición $6,8 \pm 0,258 ; 8,2 \pm 1,15 ; 8,2 \pm 1,13 ; 8,3 \pm 0,823$ y $8,1 \pm 0,80 \mathrm{~mm}$ (Figura 3).

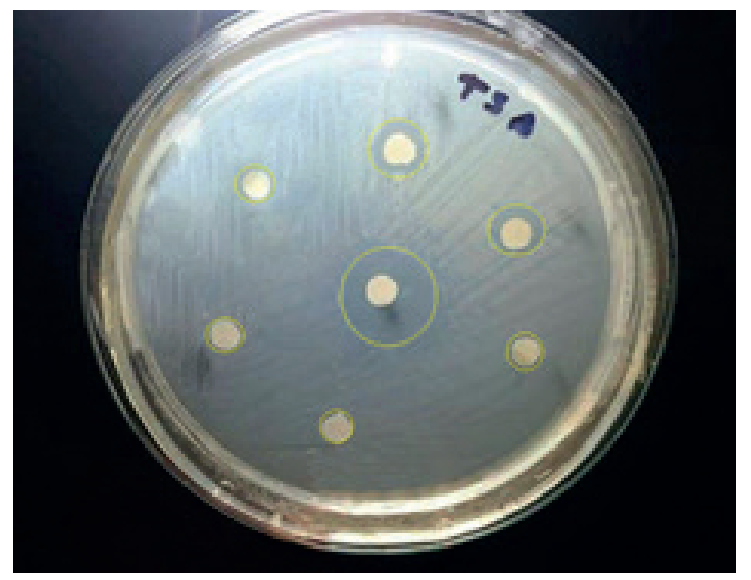

Figura 3. Halos de inhibición del extracto de S. rebaudiana a diferentes concentraciones sobre $S$. sanguinis en agar TSA

En la concentración de $15 \mathrm{mg} / \mathrm{ml}$ los diámetros de los halos están dispersos entre el $25 \%$ y $50 \%$, mientras que en las concentraciones de $50 \mathrm{mg} / \mathrm{ml}, 60 \mathrm{mg} / \mathrm{ml}$ y 120 $\mathrm{mg} / \mathrm{ml}$ los diámetros de los halos están dispersos equitativamente entre el $25 \%$ a $50 \%$ y $50 \%$ a $75 \%$. En la concentración del $30 \mathrm{mg} / \mathrm{ml}$ los diámetros de los halos están más dispersos entre el 50\% y 75\% de la muestra (Figura 4).

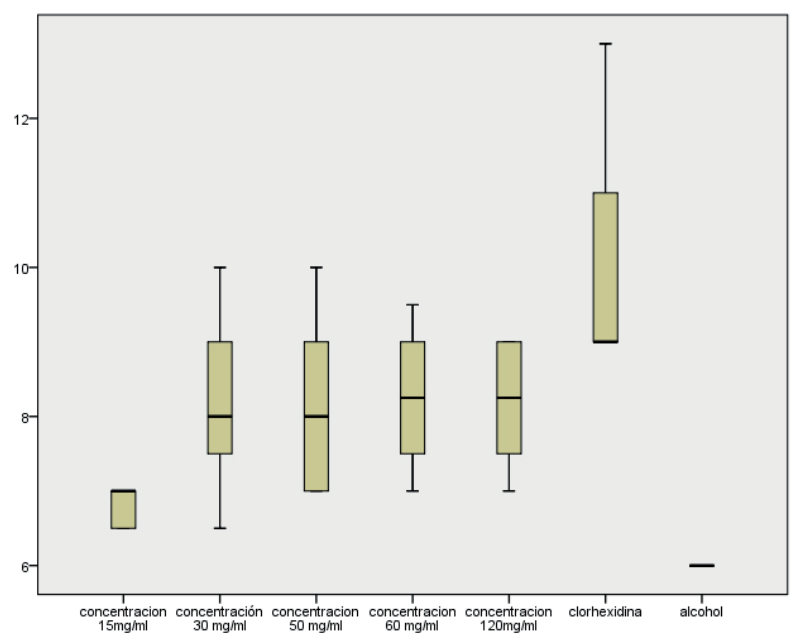

Figura 4. Resultados de los halos de inhibición en $\mathrm{mm}$ sobre $\mathrm{S}$. sanguinis causados por las diferentes concentraciones de Stevia rebaudiana, como de los controles positivo y negativo

\section{Discusión}

Actualmente se investigan numerosas plantas con el objetivo de encontrar en ellas propiedades antimicrobianas que luego puedan ser utilizados medicinalmente ${ }^{16} . S$. rebaudiana es una planta que es utilizada en la medicina tradicional por sus múltiples propiedades. En la presen- te investigación se buscó determinar el efecto antibacteriano "in vitro" del extracto de $S$. rebaudiana frente a $S$. sanguinis y $A$. viscosus. No se encontró un trabajo sobre el efecto antibacteriano del extracto de $S$. rebaudiana sobre las cepas de $S$. sanguinis pero en el estudio de Pérez ${ }^{17}$ al evaluar el efecto antibacteriano del extracto etanólico de hojas frescas de la $S$. rebaudiana sobre el $S$. mutans, con seis concentraciones en etanol de $70^{\circ}$ y seis concentraciones en etanol de $30^{\circ}$, concluyó que el extracto etanólico de $S$. rebaudiana posee efecto antibacteriano sobre $S$. mutans, bacteria del mismo género al cual pertenece el $S$. sanguinis.

Gamboa et al. ${ }^{18}$ en su estudio acerca de la actividad antibacteriana de los extractos de las hojas de $S$. rebaudiana sobre microorganismos cariogénicos, como las bacterias del género Streptococcus y Lactobacillus, determinó un mejor resultado en la inhibición del desarrollo de Lactobacillus, pero también pudo inhibir al género Streptococcus, al cual pertenece el $S$. sanguinis.

Vitery et al. ${ }^{19}$ en su estudio que evaluó la actividad inhibitoria de la $S$. rebaudiana sobre el $L$. acidophillus y $S$. mutans, comprobaron que esta tiene actividad antibacteriana sobre estos géneros. También Tovar et al. ${ }^{20}$ evaluaron la actividad antimicrobiana del extracto de Stevia en comparación con el xilitol sobre el $S$. mutans obteniendo como resultado que la $S$. rebaudiana presenta mayor efecto antimicrobiano que el xilitol.

Jayaraman et al. ${ }^{21}$ evaluaron las actividades antimicrobianas y antitumorales del extracto de $S$. rebaudiana, comparando el efecto de cuatro extractos disueltos en diferentes solventes como, acetato de etilo, acetona, cloroformo y agua, contra Staphylococcus aureus, Salmonella typhi, Eschericha coli, Bacillus subtilis, y Vibrio cholerae, utilizando el método de difusión de agar con pozos. Así también se evaluó la actividad antifúngica sobre Candida. albicans, Criptococcus neoformans. Tricophyton mentagrophytes y Epidermopython, siendo el extracto de acetona el que presentó mayor actividad frente a Gram positivas, por lo que este mismo efecto se podría dar sobre $S$. sanguinis y $A$. viscosus ya que son Gram positivos los dos microorganismos.

Lin et al. ${ }^{22}$ comprobaron la acción antibacteriana y antitumoral de los análogos de esteviósido, los compuestos sintetizados fueron más activos contra bacterias Gram positivos como el Bacillus subtilis. El estudio realizado evaluó el efecto antibacteriano del extracto de $S$. rebaudiana sobre $A$. viscosus, cuyo resultado fue que a la concentración de 30 y $50 \mathrm{mg} / \mathrm{ml}$ presentan sensibilidad límite según la escala de Duraffort, pero no se encontró estudios donde lo hayan enfrentado contra esta bacteria. Mención aparte, se deben realizar más estudios utilizando diferentes procedimientos, sean in vivo o in vitro, ya que a la luz del conocimiento actual, Stevia rebaudiana, sería un buen prospecto para su posible uso en colutorios y pastas dentales, ya sea por su efecto antibacteriano como por su condición de edulcorante.

Se concluye que bajo las condiciones de los procedimientos realizados en los ensayos, el extracto hidro-alco- 
holico de Stevia rebaudiana tiene un efecto antibacteriano in vitro sobre $A$. viscosus, para las concentraciones de 30 y $50 \mathrm{mg} / \mathrm{ml}$, no existe efecto antibacteriano in vitro del extracto de $S$. rebaudiana sobre $S$. sanguinis.

\section{Agradecimientos}

Al Vicerrectorado de Investigación de la Universidad Nacional Mayor de San Marcos por el financiamiento económico parcial, para el desarrollo del presente estudio, así como al Instituto de Investigación de la Facultad de Odontología de la Universidad Nacional Mayor de San Marcos por el apoyo en la ejecución del estudio. Así también a la Srta. Yuli Ramos P. por su apoyo en la recolección de bibliografía y tipeo del artículo.

\section{Referencias bibliográficas}

1. Minsa. Salud Bucal. Ministerio de Salud del Perú;2002 (Citado el 05 de noviembre de 2017) Disponible en : https://www.minsa.gob.pe/portalweb/06prevencion/ prevencion_2.asp?sub5=13

2. OMS. Salud Bucodental. Organización Mundial de la Salud; 2012 (Citado el 06 de noviembre del 2017) Disponible en: www.who.int/mediacentre/factsheets/fs318/ es/

3. Sitkiewicz I. How to become a killer, ori s it all accidental? Virulence strategies in oral Streptococci. Mol Oral Microbiol. 2017:1-12. doi: 10.1111/omi.12192.

4. Kreth J, Giacaman R, Raghavan R, Merrit J. The road less traveled- defining molecular commensalism with Streptococcus sanguinis. Mol Oral Microbiol. 2017;32(3):181196.

5. Ramos PD, Brañez K. Streptococcus sanguinis y Actinomyces viscosus bacterias pioneras en la formación de biofilm dental. Kiru. 2016;13(2):179-184.

6. Könönen E, Wade WG. Actinomyces and related organisms in human infections. Clin Microbiol Rev. 2015;28(2):419-422.

7. Gonzáles A, Martínez T, Alfonzo B, Rodríguez JA, Morales A. Caries dental y factores de riesgo en adultos jóvenes: Distrito Capital, Venezuela. Rev Cubana Estomatol 2009;46(3):30 -37.

8. Szpunar S, Eklund SA, Burt BA. Sugar consumption and caries risk in schoolchildren with low caries experience. Community Dent Oral Epidemiol.1995;23(3):142-146.

9. Campos C. Etiología de la caries, Streptococcus mutans, capacidad buffer salival y tipo de dieta. Revista ADM. 1985;42:43-50.

10. Liébana J. Microbiología oral. 2da ed. Madrid: McGraw-Hill -Interamericana; 2002. Capítulo 33, Bacilos Grampositivos anaerobios facultativos de interés oral; p. 345-347.
11. Bascones A. Tratado de odontología (tomo I). 4ta ed. Barcelona: Ediciones Avances; 2001. Capítulo 5, Microbiología oral; p. 682-694.

12. Escribano M, Matesanz P, Bascones A. Pasado, presente y futuro de la microbiología de la periodontitis. Av Periodoncial. 2005;17(2):79-87.

13. Bazalar D. Choquesillo F. Milla H, Herrera O, Félix M. Acción inhibitoria de crecimiento de la asociación de los extractos acuosos de Erythroxylum novogranatense (morrys) Var. Truxyllense (rusby) y Plantago major (llantén) frente a bacterias y hongos. Ciencia e investigación UNMSM. 1998;1(2):58-61.

14. Moromi H, Martínez E, Villavicencio J. Efecto del té verde en la formación de la placa bacteriana 'por Streptococcus mutans. Odontol. Sanmarquina. 2006;9(2):15-17.

15. EDAC. Manual técnico de producción de Stevia - agricultura ecológica [Internet].Cajamarca: EDAC;2008 [citado 3 de noviembre de 2016]. Disponible en: http:// agricultura-ecologica.servidor-alicante.com/documentos-agricultura-ecologica/Agricultura-Ecologica-Manual-tecnico-de-produccion-de-Stevia.pdf.

16. Moromi NH, Martinez CE, Ramos PD. Antibacterianos naturales orales: Estudios en la Facultad de Odontología de la Universidad Nacional Mayor de San Marcos. Odontol Sanmarquina. 2009;12(1):25-28.

17. Pérez Guevara SP. Efecto antibacteriano in vitro del extracto etanólico de Stevia rebaudiana sobre el Streptococcus mutans ATCC 25175. [tesis para optar el título de Cirujano Dentista]. [Trujillo]: Universidad Nacional de Trujillo; 2013. 70p.

18. Gamboa F, Chaves M. Antimicrobial potential of extracts from Stevia rebaudiana leaves against bacteria of importance in dental caries. Acta Odontol Latinoam. 2012;25(2):171-175.

19. Vitery S, Escribano V, Gamboa J, Chavarria B, Gómez S. Actividad inhibitoria de la Stevia rebaudiana sobre el Lactobacillus acidophillus y el Streptococcus mutans; Rev Nal Odo UCC. 2010;6(10):57-64.

20. Tovar G, Cupé A. Actividad antimicrobiana de la Stevia en comparación con el xilitol, frente a los Streptococcus mutans - un estudio in vitro. Rev OACTIVA UC Cuenca. 2016;1(2):51-54.

21. Jayaraman S, Manoharan MS y Lllanchezian S. In-vitro antimicrobial and antitumor activities of Stevia rebaudiana (Asteraceae) leaf extracts. Trop J Pharm Res. 2008;7(4):1143-1149.

22. Lin LH, Lee LW, Sheu SY,Lin PY, Study on the Stevioside, Steviol, and Isosteviol 19-Alkyl Amide Dimers: Synthesis and cytotoxic and antibacterial activity. Chem Pharm Bull. 2004;52(9):1117-1122. 
